\title{
Measurement of single and multiple cosmic muons at high altitudes with the MEV telescope
}

\author{
Francesco Riggi ${ }^{1 a b}$, Danilo Bonanno ${ }^{\text {ab, }}$ Giuseppe Gallo ${ }^{\text {ac }}$, Paola La Rocca ${ }^{\text {ab }}$, Fabio \\ Longhitano $^{\mathrm{b}}$, Domenico Lo Presti ${ }^{\mathrm{ab}}$ and Chiara Pinto ${ }^{\mathrm{ab}}$ \\ ${ }^{a}$ Department of Physics and Astronomy E.Majorana, University of Catania, Via S.Sofia 64, Catania, Italy \\ ${ }^{b}$ INFN, Sezione di Catania, Via S.Sofia 64, Catania, Italy \\ ${ }^{c}$ INFN, Laboratorio Nazionale del Sud, Via S.Sofia 62, Catania, Italy \\ E-mail: Francesco.Riggi@ct.infn.it
}

\begin{abstract}
An experimental investigation of single and multiple muon tracks detected by the MEV (Muography of Etna Volcano) cosmic ray telescope has been carried out during a commissioning phase of the project. The MEV telescope, which is based on three $1 \times 1 \mathrm{~m}^{2}$ tracking planes segmented into scintillator strips with wavelength fibers and PMT readout, has been installed at an altitude of about $3100 \mathrm{~m}$ a.s.l., in front of the North-East Etna crater, since the summer of 2017. The analysis of a first sample of data, taken during a period of approximately two months in 2017, has been carried out to investigate the angular distribution of cosmic muons both from the open sky side and through a large solid rock thickness (Etna side). Due to the orientation of the MEV telescope even the anisotropy caused by the East-West effect could be observed, obtaining the East-West asymmetry parameter in small steps of the zenithal angle, from about $45^{\circ}$ to $70^{\circ}$.
\end{abstract}

European Physical Society Conference on High Energy Physics - EPS-HEP2019 -

10-17 July, 2019

Ghent, Belgium

\footnotetext{
${ }^{1}$ Speaker

(c) Copyright owned by the author(s) under the terms of the Creative Commons

Attribution-NonCommercial-NoDerivatives 4.0 International License (CC BY-NC-ND 4.0).
} 


\section{Introduction}

This contribution reports a preliminary study of single and multiple muon track events, obtained during a commissioning phase of the MEV (Muography of Etna Volcano) cosmic ray telescope [1]. This facility has been installed in 2017 on top of the Etna Volcano, at an altitude of about 3100 m a.s.l., in a harsh environment, close to the active North-East crater. Although this facility was especially designed to study the mountain inner structure by the muography technique and to check its evolution during the years, we also evaluated the opportunity of carrying out physics investigations of the cosmic ray radiation, taking into account the peculiar location of this facility, i.e. its altitude and close proximity to a large solid rock structure.

In this analysis we investigated the zenithal angular distributions of cosmic muons, exploiting a range from about $45^{\circ}$ down to the horizontal, both from the Etna side (spanning a large thickness of solid rock) and from the open sky side. Due to the geographical orientation of the telescope axis, an investigation of the East-West asymmetry was also carried out in a wide zenithal angular range, in small steps from about $45^{\circ}$ to $70^{\circ}$. Other physics topics of interest also include the study of very inclined (nearly horizontal) cosmic muons and the observation of muon bundles. Preliminary results concerning two-track events measured both from the Etna side and from the open sky side were already published [2]. For this analysis a dataset collected in about 54 days data taking was considered. Such data were obtained during the commissioning run of the MEV telescope (Summer 2017), where the first tomographic images of the top craters of the Etna volcano were also produced.

\section{The experimental setup}

The MEV tracking telescope was built at the Department of Physics and Astronomy E.Majorana of the University of Catania [1]. The detector makes use of six (three along $\mathrm{X}$ and three along Y) position-sensitive planes, each with a sensitive area of $1 \mathrm{~m}^{2}$, with the two external planes at a distance of about $1 \mathrm{~m}$. Fig. 1 shows a layout of the setup. In its present status the telescope is installed inside a small container to protect it from the weather conditions, close to the Etna North-East crater, at about 3100 m altitude. Each of the six planes hosts a set of 99 scintillating strips (with a nominal size of $1 \times 1 \times 100 \mathrm{~cm}^{3}$ ), coated with white paint, with two embedded WLS fibers (1 mm diameter) to transport the photons to multi-anode 64 channels Hamamatsu H8500 PhotoMultipliers. The readout architecture of the telescope makes use of a channel reduction strategy, aimed at optimizing the number of required electronics channels and power consumption by a proper coupling of the WLS fibers (two per strip). During acquisition, data are stored in a local SD card memory. 


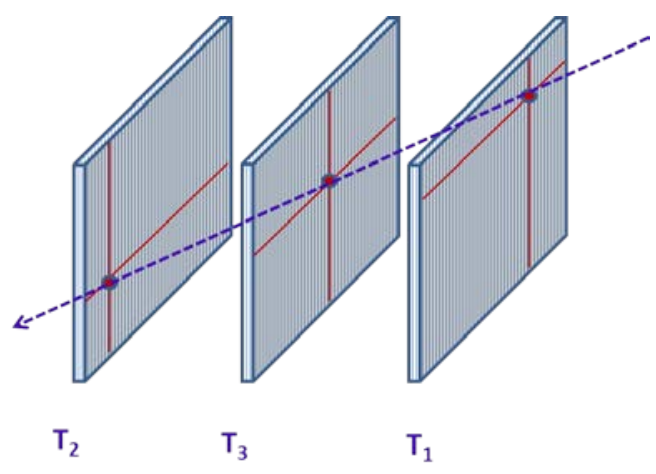

Fig.1: Layout of the MEV telescope, with its three $1 \mathrm{x} 1 \mathrm{~m}^{2}$ tracking planes, each including two physical planes, one along the $\mathrm{X}$ - and the other along the Y-direction, segmented into scintillator strips with WLS fibers and PMT readout.

The detector is also equipped with a 4G LTE wireless router. When the network signal is available, the data are sent to a cloud storage at the Department of Physics at a rate depending on the muon flux and trigger conditions. Additional details on the architecture and performance of the MEV telescope may be found in Ref.[1]. Due to the size of the detection planes and the relative distance between the two outermost planes (about $1 \mathrm{~m}$ ), a field of view of about $45^{\circ}$ is obtained for each of the two sides if the track has to cross all the planes. An average efficiency of $80 \%$ has been estimated for each plane, which led to an overall detection efficiency of about $28 \%$ for the telescope.

\section{Experimental results and discussion}

During the commissioning phase of the project, events were collected by imposing a trigger condition given by any two-fold coincidence between the three $\mathrm{X}-\mathrm{Y}$ logical planes. For this analysis, in order to have a better track reconstruction, only the events with a proper hit in all three X-Y planes were retained, which allows to reconstruct tracks with zenithal angles from about $45^{\circ}$ to the horizontal $\left(90^{\circ}\right)$. Due to the geometry of Etna and the location of the telescope, it can be inferred that the solid rock structure of the volcano is roughly confined in the angular region between $70^{\circ}$ and $90^{\circ}$, whereas the top field of view region (from $45^{\circ}$ to $70^{\circ}$ ) is free of obstacles and is comparable to what is observed from the opposite side (open sky). Taking into account short periods for calibration, maintenance and tests, a dataset corresponding to about 54 days was correctly processed and analyzed for the purpose of the present investigation. A number of about $96 \mathrm{M}$ events was collected with the trigger conditions defined above. Selection of events with a proper information on all three X-Y planes resulted in a number of good tracks of about $9.6 \mathrm{M}$. Among these, $4.4 \mathrm{M}$ were coming from the Etna side, while $5.2 \mathrm{M}$ from the opposite (back) side of the telescope. 


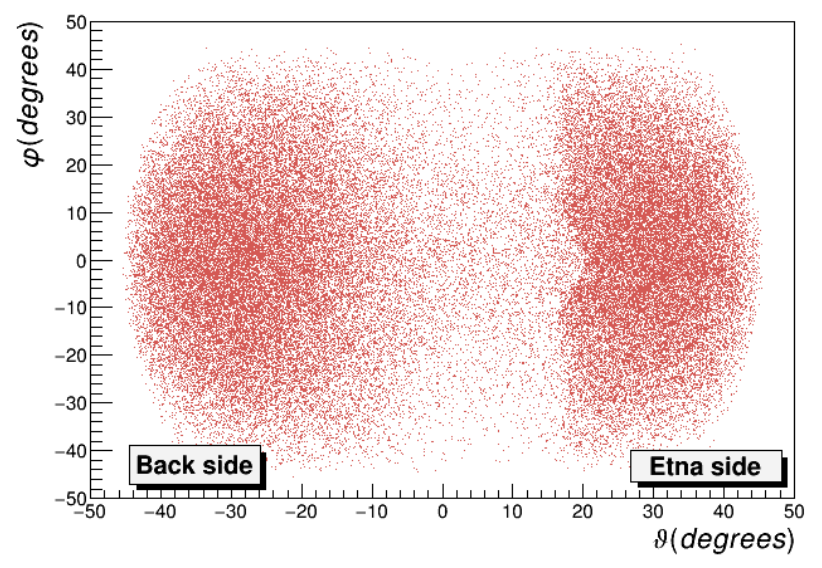

Fig.2: Two-dimensional scatter plot of a small subset of tracks in the $(\theta, \varphi)$ plane, where $\theta$ is the angle with respect to the horizontal $(\theta=0)$ and $\varphi$ is the azimuthal angle, with respect to the telescope axis.

Fig. 2 shows a 2D map of the reconstructed tracks. It may be easily seen the effect of the shadow of Mount Etna on the right side of the picture. Angular distributions were corrected for the geometrical acceptance of the telescope by Monte Carlo simulations. The acceptancecorrected zenithal angular distribution from the open sky side is shown in Fig.3, compared to a fit carried out with $A \cdot \cos ^{n} \Theta$, where $\Theta$ is the zenithal angle, obtaining a value of $n=1.765 \pm$ 0.003. It must be observed that values smaller than the usual $n=2$ dependence have been widely reported for muon momenta in the range $1-10 \mathrm{GeV} / \mathrm{c}$ [3]. Since the telescope axis is directed at about $20^{\circ}$ from the East-West direction, it was possible to select azimuthal angles around this direction, in order to investigate the East-West asymmetry at various zenithal angles. The result is shown in Fig.4, where an azimuthal window of $\pm 10^{\circ}$ around the East-West direction was selected. As it is seen, asymmetry factors up to $18 \%$ were observed for large zenithal angles, with an increasing trend from the horizontal direction to smaller zenithal angles. An analysis of twotrack events was also carried out, identifying in the large data sample of $9.6 \mathrm{M}$ tracks a subset of only 64 events ( 35 from the back side and 29 from the Etna side). Most of these identified two tracks have a small relative angle, as it is shown in Fig.5, which reports the distribution of this quantity, after correcting for the detector acceptance by the event mixing method. Further details on this analysis are reported in our recent paper [2]. An average ratio of the order of $10^{-5}$ was estimated with respect to single track events. This value takes also into account the efficiency for two track events. Although the statistics of the present dataset is limited, due the very low abundance of such events, which would require years of data taking or much larger detector areas, a first comment to these results is concerned just with the observed rate of these shower events, as identified by two correlated tracks. For muons traversing several hundred meters of solid rock, as it is the case for large zenithal angles $\left(\Theta>70^{\circ}\right)$, the observed rate was of the order of 0.1 day with this experimental setup. Such estimate may be used to discuss the possibility to search for possible upward-going showers originating from the opposite side. 


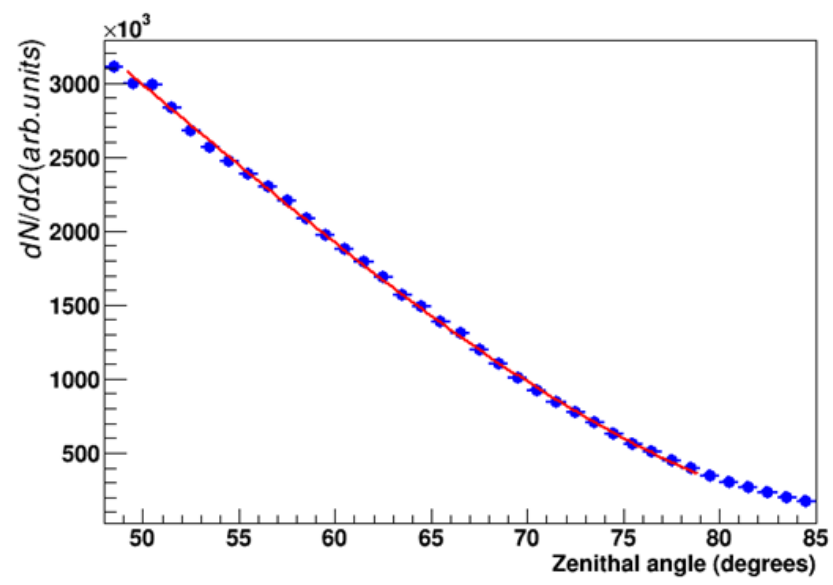

Fig.3: Zenithal angular distribution of the single tracks, from about $45^{\circ}$ to large zenithal angles, compared with the fit carried out by the function $A \cdot \cos ^{n} \Theta$.

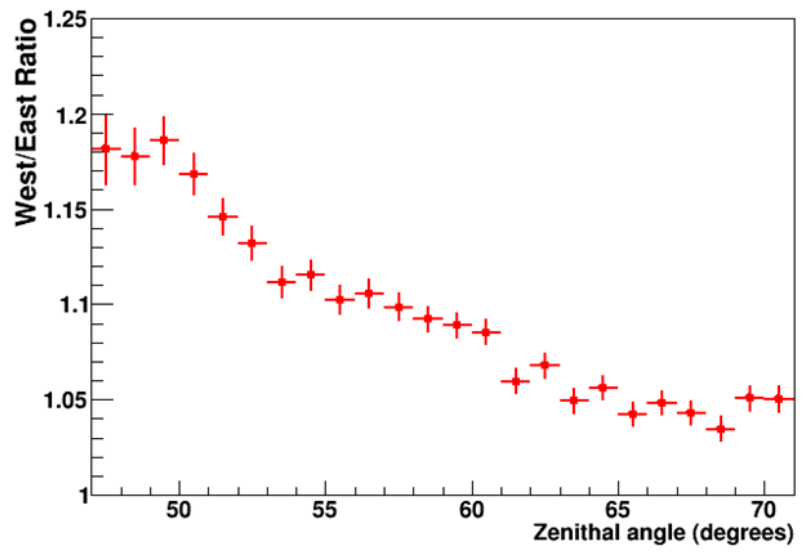

Fig.4: Ratio between the yields measured along the West and the East directions, as a function of the zenithal angle.

\section{Conclusions}

A preliminary analysis of a first set of measurements of inclined muon tracks was carried out with the MEV scintillator-based tracking telescope, installed at an altitude of $3100 \mathrm{~m}$ a.s.l. in front of the North-East crater of the Etna volcano. A commissioning data taking period of approximately two months allowed the reconstruction of $9.6 \mathrm{M}$ single track events, with an average measured rate of about $2 \mathrm{~Hz}$. These events are presently used for muon tomographic imaging of mount Etna. 


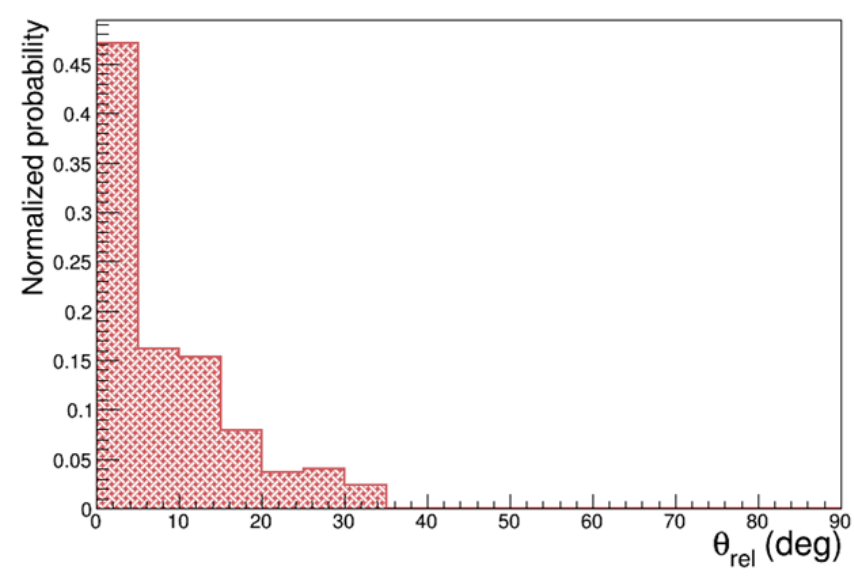

Fig.5: Distribution of the relative angle measured for two-tracks events, after correction by the geometrical acceptance in the telescope.

For the purpose of the present investigation, zenithal angle distributions were extracted and compared with a dependence $A \cdot \cos ^{n} \Theta$. East-West asymmetry effects were also investigated in small steps of the zenithal angle. A search for multiple track events was also performed in the same dataset, resulting in a small sample of events which were identified as two-track events, either coming from the Etna side or from the opposite (open sky) side. Upgrade activities are in progress to complement the MEV telescope with additional detectors and electronics contributing to better improve the selection of the incoming direction for tracks very close to the horizontal, namely a measurement of the time-of-flight between the two outermost detectors (although on a limited baseline) and the addition of a Cerenkov detector to provide directionality information [4].

\section{References}

[1] D.Lo Presti et al., The MEV Project: Design and testing of a new high-resolution telescope for muography of Etna volcano, Nuclear Instruments and Methods in Physics Research A904(2018)195.

[2] D.Bonanno et al., Measurement of nearly horizontal cosmic muons at high altitudes with the MEV telescope, Eur.Phys.J. Plus 134(2019)281.

[3] P.K.F. Grieder, Cosmic Rays at Earth, Elsevier Science, Amsterdam, 2001.

[4] D.Lo Presti et al., Feasibility study of a new Cerenkov detector for improving volcano muography, Sensors 19(2019)1183. 